\title{
ASO Video Abstract: Microsatellite Instability and the Effectiveness of Adjuvant Treatment in pT1N1 Gastric Cancer-A Multi-cohort Study
}

\author{
Namkee Oh, MD ${ }^{1}$, Hyunki Kim, MD, $\mathrm{PhD}^{2}$, Kyoung-Mee Kim, MD, $\mathrm{PhD}^{3}$, Jae-Ho Cheong, MD, $\mathrm{PhD}^{4}$, \\ Jeeyun Lee, MD, $\mathrm{PhD}^{5}$, Sung Hoon Noh, MD, $\mathrm{PhD}^{4}$, Tae Sung Sohn, MD, PhD ${ }^{1}$, Yoon Young Choi, MD, PhD ${ }^{4,6}$, and \\ Ji Yeong An, MD, PhD \\ ${ }^{1}$ Department of Surgery, Samsung Medical Center, Sungkyunkwan University School of Medicine, Seoul, Korea; \\ ${ }^{2}$ Department of Pathology, Yonsei University College of Medicine, Seoul, Korea; ${ }^{3}$ Department of Pathology and \\ Translational Genomics, Samsung Medical Center, Sungkyunkwan University School of Medicine, Seoul, Korea; \\ ${ }^{4}$ Department of Surgery, Yonsei University College of Medicine, Seoul, Korea; ${ }^{5}$ Division of Hematology-Oncology, \\ Department of Medicine, Samsung Medical Center, Sungkyunkwan University School of Medicine, Seoul, Korea; \\ ${ }^{6}$ Department of Surgery, CHA Ilsan Hospital, CHA University School of Medicine, Ilsan, Korea
}

Our study aimed to investigate the usefulness of microsatellite status in predicting prognosis and response to adjuvant treatment in pT1N1 gastric cancer (https://doi. org/10.1245/s10434-021-10084-0). MSI status may not be a prognostic factor for tumor recurrence or a predictor of response to adjuvant treatment in pT1N1 gastric cancer patients. 